\title{
La Cinthia di Enea Silvio Piccolomini. Note di lettura
}

\section{Stefano Pittaluga}

\section{OpenEdition}

\section{Journals}

Edizione digitale

URL: http://journals.openedition.org/cei/90

DOI: $10.4000 /$ cei.90

ISSN: 2260-779X

\section{Editore}

UGA Éditions/Université Grenoble Alpes

\section{Edizione cartacea}

Data di pubblicazione: 15 octobre 2011

Paginazione: $37-44$

ISBN: 978-2-84310-207-3

ISSN: 1770-9571

Notizia bibliografica digitale

Stefano Pittaluga, «La Cinthia di Enea Silvio Piccolomini. Note di lettura», Cahiers d'études italiennes [Online], 13 | 2011, online dal 15 avril 2013, consultato il 28 mars 2021. URL: http:// journals.openedition.org/cei/90 ; DOI: https://doi.org/10.4000/cei.90 


\title{
LA CINTHIA DI ENEA SILVIO PICCOLOMINI. NOTE DI LETTURA
}

\author{
Stefano Pittaluga \\ Université de Gênes
}

La Cinthia, raccolta di ventitré carmi elegiaci, fu composta da Enea Silvio Piccolomini fra il I423 e il I442 nell'ambito del vivace e raffinato entourage intellettuale formatosi presso lo Studium di Siena negli anni Venti e Trenta del Quattrocento. Un ambiente effervescente nel quale la riscoperta dell'elegia d'amore latina e della letteratura epigrammatica favorì un processo di aemulatio che si concretò in una stagione di produzione letteraria di argomento erotico (ben diversa da quella dell' "Umanesimo civile» fiorentino). Protagonisti principali di tale intensa stagione furono letterati quali Antonio Beccadelli con l'Hermaphroditus, Giovanni Marrasio con l'Angelinetum e lo stesso Enea Silvio Piccolomini .

Dopo la perduta Nimphilexis, la Cinthia si configura infatti come una prima riflessione di Enea Silvio sul tema della letteratura d'amore, destinata a maturare e a diversificarsi in forme letterarie più complesse, quali la commedia Chrysis, la novella epistolare Historia de duobus amantibus e la breve Epistula de amoris remedio $^{2}$. Si tratta di un percorso intellettuale che, pur nella costante prospettiva della docta varietas ${ }^{3}$, conduce da una prevalente presenza del modello properziano nella Cinthia a un recupero, almeno sul piano della macrostruttura, della scansione ovidiana rappresentata da Amores, Heroides, Ars amatoria, Remedia amoris ${ }^{4}$.

I. Sull'ambiente culturale senese nel primo Quattrocento si veda G. Resta (ed.), Johannis Marrasii Angelinetum et carmina varia, Palermo, Centro di studi filologici e linguistici siciliani, I976, pp. II-I5; G. Albanese, "Civitas Veneris". Percorsi dell'elegia umanistica intorno a Piccolomini», in Poesia umanistica latina in distici elegiaci, a cura di G. Catanzaro e F. Santucci, Assisi, Accademia Properziana del Subasio, I999, pp. I25-I64 (I27-I33).

2. G. Albanese, «Civitas Veneris», op.cit., p. I34.

3. Si veda P. Galand-Hallyn, «Pie II, poète élégiaque dans la Cinthia», in Pio II e la cultura del suo tempo, a cura di L. Rotondi Secchi Tarugi, Milano, Guerini e Associati, I99I, pp. I05-II7; Ead., «La poétique de jeunesse de Pie II : la Cinthia», Latomus, 52-4, 1993, pp. 875-896.

4. G. Albanese, «Civitas Veneris», op.cit., p. I52 e passim. 
Il testo della Cinthia, tramandato dal solo manoscritto autografo Vat. Chig. H IV $135^{5}$, fu pubblicato per la prima volta da Giuseppe Cugnoni nel 1883 in un'edizione che risulta non solo discutibile per molti aspetti, ma anche lacunosa, perché l'editore ha omesso quattro elegie, censurate pudoris causa ${ }^{6}$. Nel 198I Rino Avesani pubblicò le quattro elegie mancanti e propose correzioni ed emendamenti all'editio princeps ${ }^{7}$. Infine il testo completo delle ventitré elegie che compongono la raccolta fu pubblicato da Adrian Van Heck nel 1994 in un'edizione critica che rappresenta oggi il testo di riferimento per le ricerche sulla Cinthia ${ }^{8}$.

Propongo qui di seguito alcune note di lettura sul testo della Cinthia, che presuppongono la relativamente abbondante letteratura critica fiorita negli ultimi venticinque anni attorno alla raccolta elegiaca di Enea Silvio Piccolomini: rinvio per questo all'esauriente profilo bibliografico tracciato da Jean-Louis Charlet nel $2007^{9}$.

Il significato proemiale e programmatico dell'elegia I (In Cinthiam) è stato più volte indagato ${ }^{10}$ : ne emerge infatti la duplice prospettiva letteraria che percorre la raccolta elegiaca, vale a dire l'immagine della donna amata come fonte e ispirazione della poesia (vv. 3-4: Tu mihi das ipsas scribenda in carmina vires, / tu facis ingenium, tu facis eloquium), e - quasi una ricompensa - il potere della poesia di procurare fama imperitura alla donna cantata (vv. 7-8: Summa tibi, fateor, debentur premia: summo / te quoque, si liceat, carmine ad astra feram) ".

Sono temi elegiaci derivati principalmente da Properzio, il quale, e non solo per il titolo, è certamente il modello principale della Cinthia ${ }^{12}$, con buona pace di Van Heck, che tende a sottolinearne piuttosto gli imprestiti

5. R. Avesani, «Poesie latine edite e inedite di Enea Silvio Piccolomini», in Miscellanea Augusto Campana, vol. I, Padova, Antenore, I98I, pp. I-26 (6). Contro l'autografia del Chigiano si pronuncia Van Heck, pp. XIV-XV (citato infra, n. 8).

6. Aeneae Silvii Piccolomini Senensis... Opera inedita, ed. G. Cugnoni, Memorie della Reale Accademia dei Lincei, Scienze morali, storiche e filologiche, serie III, 8 (1882-I883), pp. 319-686 (i Carmina sono editi alle pp. 342-370).

7. R. Avesani, «Poesie latine edite e inedite», op. cit.

8. Enee Silvii Piccolominei postea Pii PP. II Carmina, ed. A. Van Heck, Città del Vaticano, Biblioteca Apostolica Vaticana, 1994.

9. J.-L. Charlet, «État présent des recherches sur la poésie latine d'Enea Silvio Piccolomini», in Pio II umanista europeo. (Atti del XVII Convegno internazionale, Chianciano-Pienza, I8-2I luglio 2005), a cura di L. Secchi Tarugi, Firenze, Cesati, 2007, pp. 8I-88.

Io. A. Pérez Vega, C. Muńoz Relinque, «La poesía de E. S. Piccolomini: el ejemplo de Carm. I», Studi Umanistici Piceni, I4 (1994), pp. I57-I60; G. Albanese, "Civitas Veneris», op. cit., pp. I37-I39; Z. Csehy, «Il poeta dell'amore. Livelli di interpretazione in Cinthia», in Pio II umanista, op. cit., pp. 43I-439.

II. G. Albanese, "Civitas Veneris», op. cit., p. 137.

I2. A. R. Baca, "Propertian Elements in the Cinthia of A. S. Piccolomini», The Classical Journal, 67 (1972), pp. 22I-226; A. La Penna, L’integrazione difficile. Un profilo di Properzio, Torino, Einaudi, 1977, pp. 264-266 (a proposito della presenza di Properzio nella Cinthia cfr. tuttavia p. 265: «[...] l'influenza del poeta latino, anche se ben visibile in certi casi, non è vistosa come ci aspetteremmo dal titolo.»); G. Paparelli, «Properzio nella poesia 
virgiliani ${ }^{13}$. L'incidenza del modello properziano è d'altronde implicitamente segnalata dallo stesso Piccolomini nel verso collocato programmaticamente a chiusura della prima elegia (v. Io: tu mihi principium, tu mihi finis eris), che è trasparente allusione a Prop. I I2, 20: Cynthia prima fuit, Cynthia finis erit. Anche per Enea Silvio, come per Properzio, Cinzia rappresenta dunque l'inizio e la fine di un amore totalizzante: e tuttavia nel testo d'arrivo quella stessa espressione si carica di un nuovo ulteriore significato, assente nel modello; infatti il riferimento al libellus (v. 9) indica chiaramente che il medesimo concetto di principium e di finis va applicato alla raccolta stessa delle elegie ${ }^{\mathrm{I}}$ : un libellus che, così come si apre nel nome di Cinzia, nello stesso modo nel nome di Cinzia si chiude, secondo una concezione circolare e unitaria che riflette una sistemazione e una struttura che deve risalire all'autore stesso.

E infatti anche il carme 23 che chiude la raccolta, ancorché intitolato In Amorem e concepito come una invettiva contro la crudeltà del dio, è in realtà tutto giocato sulla sofferenza intima del poeta costretto da Amore ad abbandonare la città di Siena e la propria donna (vv. 37-38: at procul exagitor, procul hac me pellis ab urbe, I me procul ab domina cogis abire mea); e d'altronde il nome di Cinthia vi è ripetuto ai vv. 36, 80, 8I. A conferma infine della concezione circolare e unitaria della raccolta, è significativo che il medesimo concetto relativo alla ricompensa (o alla riconoscenza) dovuta a Cinzia per i suoi meriti d'amore, così come è definito nel carme d'apertura: (I, 7 summa, tibi, fateor, debentur premia [...] (ripreso anche in $7, \mathrm{I}$ ), ritorni nel carme di chiusura espresso in termini quasi identici, ma ora con un senso del tutto rovesciato e speculare (23, 83: hec mihi pro meritis referuntur premia tantis): è la fine di un amore, certo, ma sul piano letterario della sistemazione strutturale dell'opera, la finis rappresenta il rovesciamento speculare del principium.

D'altronde, la forte componente prevalentemente letteraria della raccolta, che induce quindi a sgombrare il campo da tentazioni di lettura in chiave meramente autobiografica ${ }^{15}$, trova conferma nei due carmi In Virgilium che trovano posto subito dopo l'elegia proemiale, quasi a segnalare un significativo, ancorché non esclusivo, modello formale (ma non contenutistico)

giovanile di Enea Silvio Piccolomini», in Properzio nella letteratura italiana. (Atti del Convegno nazionale, Assisi, I5-I7 novembre 1985), a cura di S. Pasquazi, Roma, Bulzoni, 1987, pp. 65-70.

I3. A. Van Heck, Carmina, op. cit., pp. X-XI (prolegomena).

I4. Così anche A. Pérez Vega, C. Muñoz Relinque, «La poesía», op. cit., p. I6o; Z. Csehy, «Il poeta dell’amore», op. cit., pp. 432-433.

15. A una lettura in chiave autobiografica tende G. Paparelli, Enea Silvio Piccolomini. L'umanesimo sul soglio di Pietro, Ravenna, Longo, 1978. 
che, insieme con Catullo, Orazio, Marziale, Giovenale, gli elegiaci latini e Giovanni Marrasio, entra in gioco nella complessa e raffinata rete intertestuale della Cinthia ${ }^{16}$.

Naturalmente la docta varietas, quale segno distintivo del riuso dei modelli, comporta una rielaborazione delle fonti nel testo d'arrivo secondo una prospettiva di ricezione-emulazione che non è meramente formale, ma che investe la concezione e il significato stesso della raccolta elegiaca di Piccolomini. I risultati delle ricerche condotte negli ultimi anni sui modelli della Cinthia e sui modi secondo i quali tali modelli agiscono all'interno del testo sul piano letterario ${ }^{17}$, strutturale ${ }^{18}$ e poetico ${ }^{19}$ sono tali che oggi sarebbe agevole compilare non solo un apparato delle fonti, ma anche un commento continuo che possa illustrare i complessi e raffinati rapporti che legano Enea Silvio con gli antichi e con i contemporanei.

Per offrire un minimo contributo all'indagine in questo campo, vorrei segnalare alcune allusioni che mi pare non siano state finora individuate: 9, 5 sic tibi displiceat ciliorum frictus [...] deriva da Iuv. 6, 578-9 [...] si prurit frictus ocelli / angulus; l'incipit del carme Io (Urbem qua ducit Comum via forte petebam) contamina Verg. Aen. I $4 \mathrm{OI}$ - già segnalato da Van Heck con Hor. Sat.I 9, I (Ibam forte via sacra [...]); in 16, 7-8 et tamen infelix iterum compellor eodem, / cogor et antiquo subdere colla iugo la "iunctura" compellor eodem / cogor sembra rinviare, con variazione sintattica, a Hor. Carm. II 3, 25 omnes eodem cogimur. Infine, in un articolo del 1997 Mario Martelli si interrogava con una certa compiaciuta malizia se in 19, 2I-22 (non tibi, sed multis natum se crederet et quod / das modo sola daret plurima turba sibi) Enea Silvio, "con quel quod das, intenda lasciare in dubbio se voglia far capo a un linguaggio osceno o no", ammiccando al lettore ${ }^{20}$. Da parte mia, non vedo però né ambiguità né ammiccamenti nell'espressione quod das, che è invece una normale forma del linguaggio erotico elegiaco, presente, ad esempio, in Ovidio (Ars I 674; III 579): si tratta dunque del semplice riuso di un modello antico (presente anche in 19, 28; in 20, $3 \mathrm{e}$ in 20,8$)$.

Nella raccolta si può individuare un impianto strutturale costruito su un primo gruppo (anche se non compatto) di elegie dedicate a Cinzia (I,

I6. Sull'intertestualità nella raccolta di Piccolomini si sofferma P. Galand-Hallyn, «Pie II», op. cit., pp. Io8-II3; Ead., «La poétique», op. cit., pp. 879-890.

17. G. Albanese, «Civitas Veneris», op. cit.

I8. P. Galand-Hallyn, «Pie II», op. cit.; Ead., «La poétique», op. cit.

19. J.-L. Charlet, «Éros et Érotisme dans la Cinthia d'Enea Silvio Piccolomini», in Eros et Priapus, a cura di I. De Smet e Ph. Ford, Genève, Droz, I977, pp. I-23.

20. M. Martelli, «In Aeneae Silvii Carmina annotatiunculae», Interpres, I6 (I997), pp. 245-273 (25I-252). 
$5,7)$, un nucleo grosso modo centrale di temi e personaggi diversi $(2,3,4,6$, 8,9 , IO, II, I2, I3, I4, I5, I7, I8, 20, 22) e un nucleo finale nuovamente dedicato a Cinzia (anche questo non compatto): 16, 19, 21, 23 [In amorem] (in totale ventitré testi che, come sottolinea Jean-Louis Charlet, trovano forse un significativo parallelo con i ventidue che costituiscono la Monobiblos di Properzio) ${ }^{21}$.

Ora, una stretta affinità collega le elegie 6 (In Aragnem) e 7 (In Cinthiam) ${ }^{22}$, unite sia dal tema della luna, vale a dire l'eclisse di sole (causato dalla luna) e l'eclisse di luna (o semplicemente la mancanza della luna: dum forte laboras: 7 , II), sia dal topos degli incantesimi magici che provocano le eclissi ${ }^{23}$. Tuttavia, nonostante tale evidente affinità tematica, a differenza dell' elegia 7 dedicata a Cinthia (identificata classicamente con la luna), per contro la puella protagonista della elegia 6 sarebbe sorprendentemente una non altrimenti nota Aragne. Ebbene, io credo invece che anche l'elegia 6 debba essere ricondotta a Cinzia e ne vada quindi rivendicata l'appartenenza al primo nucleo della raccolta:

\section{IN ARAGNEM}

Phebus in hesperias quamquam nondum iverit undas, vidi ego iam medios luce carere dies tum, mediam cum se niveo Latonia fratri obiicit et radios non sinit ire suos: sic mea nonnunquam miserum me fallit Aragne, ante illam cum se perfida sistit anus. o te longeve remorentur fila senecte nec vivas cupiens nec moriare volens. ut mihi siderei rapis invida lumen ocelli, sic tibi iocundum subtrahat illa merum.

Nei vv. I-6 il poeta istituisce un paragone fra il fenomeno dell'eclisse, provocato dal passaggio della luna davanti al sole (mediam...ire suos) impedendone l'irradiazione, e l'azione di una perfida anus, personaggio che si richiama alla lena degli elegiaci, insomma una mezzana che talvolta (nonnunquam) impedisce al poeta di poter incontrare l'amata fanciulla. Costei, nel testo come pubblicato concordemente da Cugnoni e da Van Heck ${ }^{24}$, ha nome Aragne, con evidente allusione al personaggio di Arachne protagonista del mito narrato da Ovidio (Met. VI 5-I45), cui indubbiamente si

2I. J.-L. Charlet, «Éros et Érotisme», op. cit., p. 3 e n. 6; Z. Csehy, «Il poeta dell'amore», op. cit., p. 433.

22. Sul testo di questa elegia cfr. A. Pérez Vega, "Notas sobre el texto de los Carmina de E. S. Piccolomini», Humanistica Lovaniensia, 39 (1990), pp. 40-47 (42-43).

23. P. Galand-Hallyn, «La poétique», op. cit., pp. 880-882.

24. Rispetto al testo di Van Heck ho ripristinato la $v$ intervocalica. 
riconduce anche il riferimento ai fila presente al v. 7. A questo proposito, Perrine Galand-Hallyn osserva che "l'image du fil est reprise par Enea Silvio dans l'imprécation qui conclut le poème, mais elle est appliquée cette fois à la lena [e non alla puella Aragne], tandis que le poète s'exclame: $\mathrm{O}$ te longeve remorentur fila senecte! L'image des Parques se profile à son tour...». Si tratterebbe dunque di una variatio audace e veramente sorprendente ${ }^{25}$.

Ma tutto torna se al v. 5, come propongo, si anticipa la collocazione della virgola, che va posta non dopo Aragne, ma dopo fallit (vv. 5-6):

sic mea nonnumquam miserum me fallit, Aragne ante illam cum se perfida sistit anus.

Così, con un enjambement non raro nei carmina di Enea Silvio, il nome di Aragne è restituito alla perfida anus e a costei si riferiscono correttamente i fila del v. 7. Per contro, soggetto di fallit è ora il possessivo mea, non più riferito ad Aragne, ma forma ellittica sostantivata in luogo di mea puella, mea domina, etc. ${ }^{26}$. Ed è questo un uso attestato nei comici (ad es. Ter. Eun. 798; Phorm. 500), ma soprattutto negli elegiaci e, significativamente, in Properzio (III 8, 22): me doceat livor mecum habuisse meam; e più volte in Tibullo (ad es. I 5, 42 [...] et narrat scire nefanda meam; II 3, 77 nunc si clausa mea est, si copia nulla videndi; II 6, 52).

Considerati dunque gli stretti rapporti contenutistici e la contiguità fra l'elegia 6 (In Aragnem) e la 7 (In Cinthiam), ritengo sia molto probabile che con l'espressione mea l'autore intenda far riferimento alla stessa Cinzia, e che quindi anche l'elegia 6 le vada rivendicata. Quanto poi al titolo In Aragnem, deve essere certamente esclusa l'equivalenza In=Contra, tenuto conto del fatto che la preposizione in seguita da nome proprio, come è presente nei singoli titoli della raccolta, sembra piuttosto indicare semplicemente il nome del personaggio protagonista, nel bene o nel male, del carme cui il titolo si riferisce.

Un analogo problema, che investe una scelta di interpunzione e l'uso del possessivo sostantivato, si rileva nel carme II (In Lisiam), di evidente tono epigrammatico:

\section{IN LISIAM}

Lisia quid redolet? sapit hec tibi Lisia mella?

et reris soli grata sit illa tibi?

25. P. Galand-Hallyn, «La poétique», op. cit., p. 882.

26. Cfr. J. B. Hofmann, La lingua d’uso latina, a cura di L. Ricottilli, Bologna, Pàtron, I980, p. 342; J. B. Hofmann, A. Szantyr, Stilistica latina, a cura di A. Traina, C. Neri, R. Oniga e B. Pieri, Bologna, Pàtron, 2002, pp. 23 I-232. 
atque tuam reputas? tamen hec in fornice vivit et tua, non vultus diligit illa tuos. alter inest oculis gratior usque suis, quem nosti et tecum ducit noctesque diesque et simulat quod se nulla puella velit. ut tibi vera loquar : meretrix est. ergo mariti iura negat multos nunc habitura procos.

Lisia si placeat, placet hec tua Lisia multis, sed magis illa colit qui magis inde dabit.

Così il testo nell'edizione Van Heck: si tratta di uno dei quattro carmi omessi da Cugnoni ed editi per la prima volta da Avesani ${ }^{27}$. Nella nota al testo Van Heck presenta i termini della questione, che riguardano i versi I e II nei quali occorre decidere se Lisia sia nome proprio maschile (Lisias, voc. Lisia), o femminile (Lisia):

Nel primo caso, se è maschile, il nome Lisia va considerato in caso vocativo ed è nei suoi confronti che il poeta rivolge i propri sarcastici versi: Lisia è quindi il nome di un marito illuso circa la fedeltà della propria puella, non nominata, ma che si rivela invece di assai facili costumi (v. 9: meretrix est): ed è stata questa la scelta testuale di Avesani, che in entrambi i versi pone Lisia fra virgole; nel secondo caso, se è femminile, Lisia risulta invece il nome della puella dalla morale facile, che va considerata soggetto in entrambi i versi in discussione, mentre è il marito illuso (al quale il poeta si rivolge in seconda persona) ad essere privo di nome proprio: ed è questa la scelta di Van Heck. A conferma della propria opinione, lo stesso Van Heck fa riferimento, in nota, al v. II («quod iudicium us. II confirmare videtur»), perché evidentemente nel nesso hec tua Lisia egli intende tua come aggettivo possessivo femminile riferito a Lisia $^{28}$.

E tuttavia, in considerazione di quanto si è osservato a proposito di mea in 6, 5, credo che anche in questo caso la analoga forma tua funga da possessivo ellittico sostantivato, sottintendendo puella; e d'altronde non mancano autorevoli attestazioni antiche per tale uso assoluto anche di tua.

27. R. Avesani, «Poesie latine», op. cit., p. 22.

28. Trascrivo qui la nota di Van Heck (p. I8, ad loc.): «Nomen Lisia(s) uel Lysia(s) non usitatum est. siue nomen feminae est (Lisia), siue uiri (Lisias), uocatiuus casus desinit in -a: Lisia. decernendum igitur est de uiro an de femina agatur, Lisia in uersiculo primo nominatiuus sit an uocatiuus. Avesani (22), primus editor huius carminis, post Lisia comma posuit: "Lisia, quid redolet? sapit hec tibi, Lisia, mella<??", putans Lisiam uirum esse. mihi magis arridet Lisiam esse feminam bene 'olentem'; quod iudicium us. II confirmare videtum. 
Ad esempio Hor. Carm. I I5, 32: non hoc pollicitus tuae [detto a proposito di Paride in riferimento a Elena].

Ritengo, in conclusione, che sia preferibile il testo come interpunto e pubblicato da Avesani, tanto più che, nell'impiego assoluto di tua, presenta le caratteristiche di lectio difficilior:

I Lisia, quid redolet? sapit hec tibi, Lisia, mella?

II Lisia, si placeat, placet hec tua, Lisia, multis.

Tutto ciò a conferma del fatto che nel campo della letteratura medievale e umanistica l'analisi dei modelli testuali - e non del mero reperimento delle fonti, oggi peraltro facilitato dagli strumenti informatici - consente non solo di individuare le strutture compositive, le soluzioni di poetica letteraria, le prospettive intellettuali presenti nei testi, che necessariamente con quei modelli entrano in emulazione, ma permette anche di verificare le modalità secondo le quali il riuso delle auctoritates (lessicali, grammaticali, sintattiche) agisce sul piano formale e sul piano tematico, fornendo così elementi decisivi per la costituzione del testo. 